\title{
Clinical Evaluation of Neck in Patients with Proprioceptive Cervicogenic Dizziness
}

Roseli Saraiva MB*

Nedison GPA

Garcia CB

\begin{abstract}
Objective: To establish a functional connection between neck physical evaluations, dizziness discomfort and image findings among subjects diagnosed with proprioceptive cervical dizziness.

Methods: After exclusion of peripheral vestibular disorders, 20 subjects with proprioceptive cervical dizziness hypothesis were selected. A Visual Analogue Scale (VAS) was used to quantify pain and vertigo. The active neck Range of Motion (ROM) and the Muscle Strength (MS) of the neck region were examined. The manipulation of vertebral bodies by the Maitland method and imaging scan were performed.

Results: A positive correlation between pain and vertigo VAS scores was found. The ROM of the cervical spine was limited and vertebral joint movement was restricted, especially at C3 and C5. No loss of MS was noticed.

Conclusions: Proprioceptive cervical dizziness is usually an exclusion diagnosis among episodic chronic vertigos. Characteristically, it is reported by patients as instability or vertigo in crises. It is directly related to the neck ache severity and worsens with neck movements. The common pattern on clinical examination includes restriction and pain during neck flexion without loss of MS. Reduction of joint mobility and pain are also observed, especially at C3 and C54 kHz.
\end{abstract}

Keywords: Tinnitus, Dizziness, Neck pain, Cervical vertebrae, Vertigo. 


\section{INTRODUCTION}

Proprioceptive Cervicogenic Dizziness (CD) is characterized by the presence of imbalance, unsteadiness, disorientation, neck pain, limited cervical range of motion (ROM), and may be accompanied by a headache ${ }^{1}$. The syndrome is attributed to abnormal afferent activity of the neck ${ }^{2}$ and is a controversial topic among specialists. Some authors suggest that proprioceptive cervical vertigo results from abnormal activity of the extensor muscles and neck joints ${ }^{3}$ and closely related to changes in cervical spine position and cervical joint movement ${ }^{4}$. The anomalous signals travel to the central nervous system (CNS) and alter postural control ${ }^{5}$. Sensorimotor control for maintaining posture is closely linked to proprioceptive afferent signals. Cervical information is derived from three reflexes involving the stabilization of the head, including the cervico-colic reflex, cervico-ocular reflex, and tonic neck reflex. These reflexes work in conjunction with vestibular and visual information, stabilizing the head, eyes, and posture ${ }^{6}$. Neck pain is a common complaint in the general population and may be viewed as a public health problem. A systematic review conducted in 2006 showed that the prevalence of neck pain in the population was $5.9 \%$ to $38.7 \%^{7}$. Within this population, some subjects experience an association between vertigo and neck pain reported that in patients who presented with more than one cause of vertigo, the incidence of cervical origin was $7.5 \%^{8}$. A prevalent complaint of patients with $C D$ is episodic disorientation and mild instability lasting from minutes to hours and worsening with cervical movement and/or increased neck pain9. Some patients with cervical pain may still report vertigo 2 , visual changes, and falls ${ }^{9}$.

A temporal relationship often exists between vertigo and neck pain, and vertigo relief is associated with decreased neck pain. Although the cervical dizziness is described by subjects who have suffered trauma, it is highly associated with pathologies of cervical spine and its related muscles Recent studies with intervertebral discs suggest the relationship between the cervical segment and balance 1. The authors studied surgically removed intervertebral discs from patients with cervical pain alone and associated with dizziness. The discs from dizzy patients had more Ruffini corpuscles than the other discs, suggesting the key role of these corpuscles in the pathogenesis of vertigo of cervical origin ${ }^{10}$.

The absence of a specific test makes the diagnosis questionable, and the symptoms are commonly attributed to other disease ${ }^{11}$. However, the great improvement produced by physiotherapeutic treatment focused on cervical proprioceptive alterations strongly suggests its etiology ${ }^{12}$. Based on these clinical observations, some authors proposed some tests in order to highlight the cervical origin of the imbalance. Pursuit with cervical torsion ${ }^{13,14}$, postural sway9 among others investigation tests are proposed to diagnose cervical dizziness. Otherwise, a recent systematic review concluded that "the most consistent diagnostic criteria were based on the concurrence of neck pain with dizziness after exclusion of other possible reasons for dizziness ${ }^{15}$. Once vestibular pathologies have been ruled out, the clinical exam of the cervical spine is important in order to confirm or exclude the diagnosis of $C D^{16}$. Some authors consider the clinical exam the gold standard to diagnose CD ${ }^{17}$. Our objective is to evaluate the functional involvement of cervical segment, its relation with dizziness discomfort and image alterations in patients diagnosed as having proprioceptive cervical dizziness.

\section{MATERIALS AND METHODS}

This is a cross-sectional study. Patients were recruited at ENT Department of our tertiary referral hospital from 2018 to 2019. The study group comprised patients clinically diagnosed with proprioceptive $C D$, treating their dizziness in the cervical ambulatory and agreed to participate in the research.

This study was approved by the Ethics Committee under the number $1,433,854$.

All patients included in the study were submitted to a complete otoneurologic evaluation including clinical examination and laboratory tests (renal function, complete blood count, fasting glucose, thyroid function, and lipogram). Video head impulse tests and/or electrophysiological tests were performed when indicated. The individuals underwent a videonystagmography with cervical torsion. The presence of nystagmus on head turning was considered suggestive of cervical involvement especially if the patient complaints of local pain and restriction to cervical movement ${ }^{9}$. The subjects were selected after excluding peripheral or central vestibulopathy.

The sample was selected according to the following inclusion and exclusion criteria.

\section{Inclusion criteria}

A. Presence at least one of the above signals:

1. History of vertigo associated with neck pain, with a temporal relationship between symptoms;

2. Nystagmus on head turning during videonystagmography;

\section{Stiffness of the neck;}

B. All the subjects were consulted, informed, and agreed to participate.

\section{Exclusion Criteria}

\section{A. Cognitive deficit}

B. Active psychiatric, neurological or rheumatologic diseases

\section{Continuous use of drugs to treat neck pain or vertigo}

D. Other otoneurologic diagnosis: migraine and associated dizziness; vestibular neuritis; vertigo of central origin; BPPV; dizziness induced by drugs; Meniere's disease; psychogenic vertigo, labyrinthine concussion, cervical arterial disfunction and whiplash associated disorder. The study group consisted of 20 patients: 17 
(85\%) women and $3(15 \%)$ men. The mean age of the group was $66.95 \pm 8.59$ years; $65.82 \pm 7.90$ years for females and $73.33 \pm 9.53$ years for males. The subjects were selected according to the described criteria and subjected to the specific evaluations. The cervical spine evaluation included manual spinal examination, joint mobility, muscle strength, active motion and displacement. All the subjects were asked to quantify the pain and related dizziness.

The study followed the steps ahead described:

1. Quantification of pain intensity and discomfort from vertigo by a visual analogue scale (VAS). Zero indicates the absence of symptoms, and 10 indicate unbearable symptoms.

2. Active range of motion (ROM) of the cervical spine with a fleximeter capable of measuring the maximum displacement angle in the sagittal, frontal, and axial planes. The patient was seated in a standard chair with the thoracic spine resting on the backrest and the hands resting on the thighs; 90 o of hip and knee flexion were maintained with the feet resting on the floor. The subject was instructed to perform active cephalic movements in three axes of displacement. The inclinometer was positioned on the head (Figure 1), and the following verbal commands were given: "put your chin on your chest", "put your head back", and "put your ear on your shoulder". Then, the patient was positioned in dorsal decubitus (DD), and the inclinometer was placed on the upper part of the head with the following verbal command: "turn your head to the right/left". The observed maximum angles were measured and compared using normality indexes.

3. Verification of the muscle strength (MS) of the cephalic region by the Kendall scale. The MS was measured in opposition to an external resistance to flexion and extension (sagittal plane, transverse axis); D/E slope (frontal plane, anteroposterior axis) and $\mathrm{D} / \mathrm{E}$ rotation (transverse plane). The scale ranged from 0 to 5 as follows: 0 - absence of contraction; 1 outline of contractions observed on muscle surface; 2 - contraction occurs but does not overcome the force of gravity; 3 - contraction occurs with complete movement against the force of gravity; 4 - a small amount of resistance is overcome; 5 - a large amount of resistance is overcome.

4. Palpation of the posterior cervical region in $\mathrm{DD}$ and verification of joint mobility (JM) of the cervical vertebrae using posteroanterior central pressure (PACP) according to the Maitland technique18. To perform the procedure, the patient is positioned in DD. The practitioner places their middle fingers on the spinous processes of the cervical vertebrae of the subject and exerts pressure in the anterior direction until meeting resistance in the tissue and/or pain (Maitland grade III)19. When the displacement is greater than expected, the joint is classified as hypermobile; mobility may be normal, or the movement may be smaller than expected (hypomobile). The patient is instructed to report any pain and/or vertigo.

5. Imaging examinations of the cervical spine: X-ray or computed tomography

Statistical Evaluation: The mean and standard deviation were calculated for the analysis of age, gender, and VAS. Nonparametric variables were assessed using the Pearson correlation coefficient, with $r>0.718$ considered significant. Parametric variables with a normal distribution confirmed by the Shapiro test were evaluated by Student's t-test, with $p<0.05$, and a $95 \%$ confidence index $(\mathrm{Cl})$ was determined. The results obtained were compared to historical data for each test.

\section{RESULTS}

The average duration of vertigo on arrival at the outpatient clinic was 9.90 years. The average VAS scores were 8.05 for neck pain and 7.47 for vertigo. A correlation was found between the severity of neck pain and vertigo; greater pain resulted in greater discomfort due to vertigo $(r=0.78)$ (Figure 2).

Instability type dizziness was reported by most patients (70\%), followed by vertiginous vertigo (25\%), and fluctuating $(5 \%)$ dizziness. When asked about the most common time of set of vertigo, 6 patients (30\%) specified the morning, $5(25 \%)$ specified the afternoon, $2(10 \%)$ specified the night, and 7 patients (35\%) specified no temporal relationship. Regarding the frequency, 2 of 3 patients reported at least 1 episode of vertigo weekly. Cervical movement was associated with worsening vertigo for 15 patients $(75 \%)$. Only 2 patients had a history of cervical trauma. The active ROM of the cervical spine was reduced in relation to normal values in all patients as the data described in (Table 1).

Only 1 of the 20 patients showed good JM. The vertebrae that presented the least mobility were $\mathrm{C} 3$ in $17(85 \%)$ subjects (of these, 14 (82\%) reported pain), followed by $\mathrm{C} 5$ in $15(75 \%)$ subjects (of these, $11(73 \%)$ reported pain), C4 and C6 in 11 (55\%) subjects, C7 in 9 (45\%) subjects, and C2 in 7 (35\%) subjects. No patient had increased mobility. All patients reported pain during some point of joint mobilization. The vertebrae most commonly associated with pain were C3 in 14 (70\%) subjects and $\mathrm{C} 5$ in 11 (55\%) subjects. The correlation between the number of vertebrae resulting in pain on palpation and the discomfort of vertigo was weak $(p=0.33)$. No relationship was found between vertigo and the two vertebrae most commonly associated with pain (Fisher's exact test $p=0.51$ ). The MS evaluation of cervical flexion movement resulted in a grade 4 classification of 13 (65\%) subjects. Grade 4 means that patients overcame a small resistance. The extension movements in $15(75 \%)$ patients were classified as MS grade 5 (overcame a large resistance). The other movements tested were classified as MS grade 5 in $12(60)$ patients. Aging-related changes were the most common imaging findings of the cervical spine as the data described in (Table 2). 

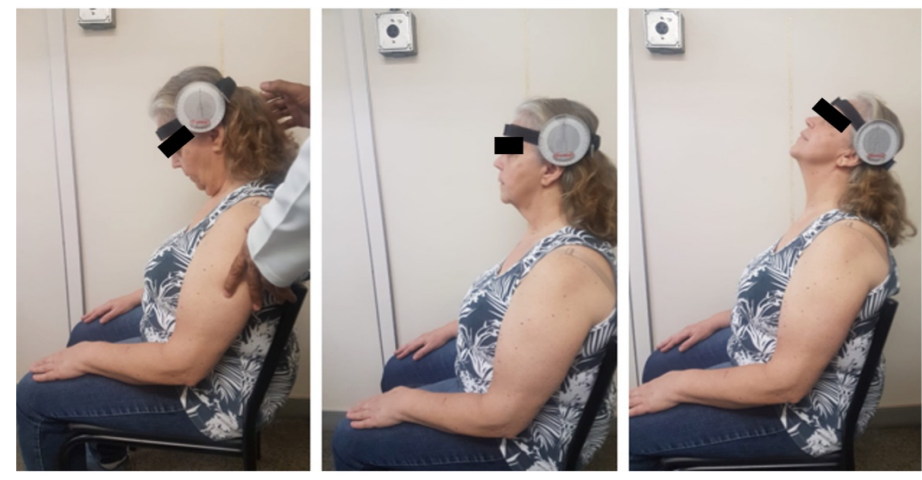

Figure 1: Patient with a fleximeter attached to the head.

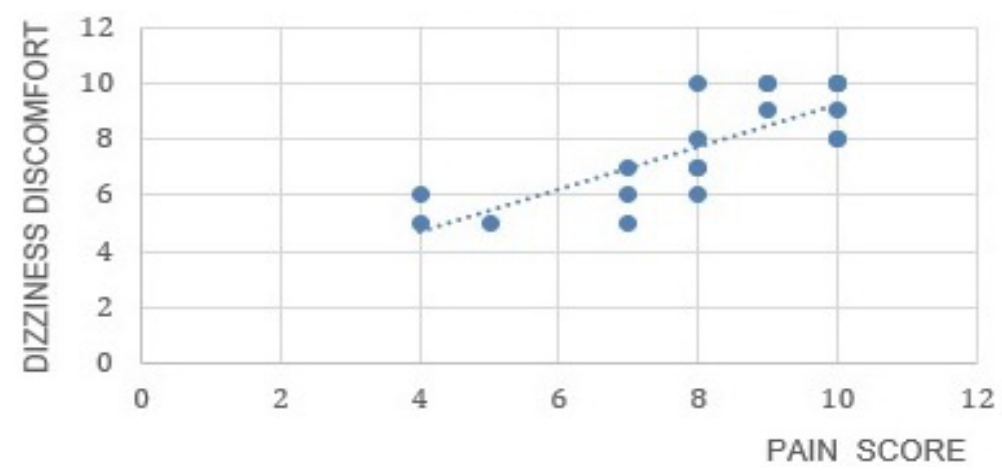

Figure 2: Visual Analogue Scale (VAS) score for pain $x$ dizziness

Table 1: ROM recorded values measured with a fleximeter.

\begin{tabular}{|c|c|c|c|c|c|c|}
\hline & FLEXION & EXTENSION & RIGHT ROTATION & LEFT ROTATION & RIGHT TILT & LEFT TILT \\
\hline NORMAL & 65 & 50 & 55 & 55 & 40 & 40 \\
\hline MEAN & 50.3 & 49.8 & 50.8 & 51.1 & 36.85 & 33.85 \\
\hline P & $<0.001^{*}$ & 0.91 & $0.05^{*}$ & $0.01 *$ & 0.01 * & $0.001 *$ \\
\hline
\end{tabular}

Table 2: Radiological findings found among patients with $C D$.

\author{
RADIOLOGICAL FINDINGS \\ Uncoarthrosis \\ Anterior marginal osteophytosis \\ Reduced joint space \\ Degenerative retrolisthesis \\ Posterior osteophytosis \\ Spondylarthrosis \\ Bone demineralisation \\ Nuchal ligament enthesopathy \\ Cervical lordosis rectification
}

PERCENTAGE (\%)
$\mathrm{n}=20$
$13(76 \%)$
$13(76 \%)$
$11(65 \%)$
$11(65 \%)$
$8(47 \%)$
$7(41 \%)$
$6(35 \%)$
$6(35 \%)$
$6(30 \%)$

These drugs reduce vestibular reflexes and predispose patients to falls, especially elderly individuals, who suffer from physiological limitations of body balance due to the ageing. To avoid diagnostic confusion in our study, all patients were treated for possible comorbidities, and participation of the peripheral vestibular system was excluded. In our diagnostic investigation we use as routine the cervical neck torsion to observe the presence of nystagmus on videonystagmography. The nystagmus from cervical segment has particularities diverse than BPPV $^{19}$. The diagnostic value of the test is the differential diagnosis the dizziness is provoked by rotation of the neck $^{16}$. 
This study of 20 patients was comprised of $85 \%$ women and $15 \%$ men. According to the literature, for all age groups, cervicalgia is a more frequent symptom in women than men ${ }^{20}$. The affected population is in approximately the fifth or sixth decades of life, and the age of our sample was slightly higher, approximately 67 years. We believe that it occurred due to the delay in receiving appropriate care, which in our case was approximately 10 years. Trauma was not found to be prevalent in the sample and may be considered a factor of lesser relevance in the onset of $C D$ in our study group. The mean intensity of the pain score was 8 , which is considered severe. Pain mainly occurred in the posterior region of the neck with lateralization following the upper part of the trapezius muscle; however, the pain did not reach the shoulder. Vertigo increased with pain perception and cervical movement, with a mean intensity of 7.47 . The correlation between pain and vertigo $(r=0.78)$ was very close to 1 (one), which is a perfect correlation. When considering the characteristics of vertigo, a predominant time of day was not found, but $30 \%$ of the subjects reported the symptom in the morning, and $25 \%$ reported vertigo in the evening. Symptoms in the morning should be attributed to the sleeping position and when in evening due to the posture during the day. The episodes also had a frequency of at least once a week in two-thirds of the sample. These characteristics allow classification of proprioceptive cervical vertigo as episodic chronic vertigo. In the vast majority of patients, vertigo presented as difficulty of postural orientation and a sensation of imbalance, which is characteristic of the instability type. Proprioceptive information is added to vestibular inputs in the multimodal neurons of the vestibular nucleus that encode and associate vestibular and proprioceptive signals. Faced with a discrepancy between expected and actual proprioceptive information, multimodal neurons do not preferentially encode the correct vestibular information, which promotes an anomalous response to the coordinates referenced by the body ${ }^{21}$. The central connections may be related to vertiginous vertigo, which is reported by a quarter of patients, leading to confusion with symptoms originating in the peripheral vestibular system. The same multimodal neurons of the vestibular nucleus promote the correspondence of vestibular and proprioceptive signals that activate the semi-circular canals and the otolithic organs involved in rotation and flexion of the spine ${ }^{22}$. Both the asymmetry of information from cervical proprioceptors and sensory conflict in the CNS, which interprets the information received as discordant with the pattern recorded on the cerebral map (efference copy), can result in the clinically described sensation of rotation 2,11. During the head rotation, the expected reafference of an intended movement may fail to correspond to the pattern of sensory input calibrated by earlier experience of head motions. Because of the pain, the intended movement falls shorter than expected because of neck muscle stiffness leading to a head motion induced misalignment of sensorimotor integration ${ }^{23}$. Excluding peripheral vestibulopathy in cases of vertigo associated with cervical pain is critical to establish the final diagnosis, treatment, and prognosis. Therefore, CD is a diagnosis of exclusion. The amplitude of cephalic movements was reduced in all patients. The most significantly compromised movement was cephalic flexion, which was affected in $90 \%$ of patients. Cephalic rotation and tilt were also affected, but extension was not an important factor in the characterization of the syndrome. We believe that the limitation of cephalic flexion is a valuable distinguishing factor in the evaluation of $C D$ due to its simple determination and great clinical significance ${ }^{23}$. As a triage test, it is an efficient way to suspect from cervical origin of the dizziness 16. Cervical mobility was restricted and painful. The C3 and C5 vertebrae were affected in the vast majority of patients. Thus, the palpation and observation of pain in these locations is informative, although not all subjects showed movement limitations in these particular vertebrae. Additionally, C3 and C5 were reported to be more painful upon movement. We could not establish a relationship between the discomfort of vertigo and the number of vertebrae involved. Muscle strength (MS) was not an important factor in cervical segment evaluation. Again, flexion was the most impaired movement in $65 \%$ of patients, yet strength was only slightly impaired and classified as grade 4 . After flexion, cephalic rotation and tilt showed little impairment, and no loss of MS in extension was observed. Therefore, no restriction of MS was found in the affected subjects. The most frequent radiological alterations were uncoarthrosis, osteophytosis, and reduction of joint space, and retrolisthesis, alterations found in $85 \%$ of individuals. We could not establish a relationship between the radiological changes and vertigo, as they are common in the age group evaluated in the study. Anyway, the high incidence of radiological alterations of spine associated to cervical pain suggests the association between the variables ${ }^{24}$. A recent research described an unusual large number of Ruffini corpuscles into cervical discs surgically removed from patients with pain and dizziness. These corpuscles may encode erroneous signals from the diseased cervical discs and muscle spindles in tense neck muscles and send it to the central nervous system leading to a sensory mismatch with other sensory information. Apparently, the abnormal neck proprioceptive input provokes the sensorial conflict and a subjective feeling of unsteadiness.

\section{CONCLUSION}

Proprioceptive cervical vertigo can be classified as a diagnosis of exclusion among the types of episodic chronic vertigo. Characteristically, it presents as episodes of instability or vertigo. It is directly related to the intensity of cervicalgia and worsens with cephalic movements. On clinical examination, restricted and painful cephalic flexion without loss of MS is a common pattern. Reduction of JM and pain are observed, especially at C3 and C5.

\section{ACKNOWLEDGEMENTS}

Funding Support: FAPESP project 2016/20176-4 


\section{CONFLICT OF INTEREST}

The authors declares no conflict of interest

\section{REFERENCES}

1. Wrisley DM, Sparto PJ, Whitney SL, Sparto PJ, Joseph FM. Cervicogenic dizziness: a review of diagnosis and treatment. J Orthop Sport Phys Ther. 2000;30:755-66.

2. Heidenreich KD, Beaudoin K, White JA. Cervicogenic dizziness as a cause of vertigo while swimming: an unusual case report. Am J Otolaryngol. 2008;29:429-31.

3. Fischer AJ, Verhagen WI, Huygen PL. Whiplash injury. A clinical review with emphasis on neuro-otological aspects. Clin Otolaryngol Allied Sci. 1997;22:192-201.

4. Reid SA, Callister R, Katekar MG. Effects of cervical spine manual therapy on range of motion, head repositioning, and balance in participants with cervicogenic dizziness: a randomized controlled trial. Arch Phys Med Rehabil. 2014;95:1603-12.

5. Bracher ES, Almeida Cl, Almeida RR. A combined approach for the treatment of cervical vertigo. J Manip Physiol Ther. 2000;23:96-100.

6. Kristjansson E, Treleaven J. Sensorimotor function and dizziness in neck pain: implications for assessment and management. J Orthop Sport Phys Ther. 2009;39:364-77.

7. Fejer R, Kyvik KO, Hartvigsen J. The prevalence of neck pain in the world population: a systematic critical review of the literature. Eur Spine J. 2006;15:834-48.

8. Ardiç FN, Topuz B, Kara CO. Impact of multiple etiology on dizziness handicap. Otol Neurotol. 2006;27:676-80.

9. L'Heureux-Lebeau B, Godbout A, Berbiche D. Evaluation of paraclinical tests in the diagnosis of cervicogenic dizziness. Otol Neurotol. 2014;35:1858-65.

10. Yang L, Chen J, Yang C. Cervical Intervertebral Disc Degeneration Contributes to Dizziness: A Clinical and Immunohistochemical Study. World Neurosurg. 2018;119:e686-e93.

11. Hain TC. Cervicogenic causes of vertigo. Curr Opin Neurol. 2015;28:69-73.
12. Bittar R, Alves NG, Bertoldo C. Efficacy of Carbon Microcoils in Relieving Cervicogenic Dizziness. Int Arch Otorhinolaryngol. 2017;21:4-7.

13. Tjell C, Rosenhall U. Smooth pursuit neck torsion test: a specific test for cervical dizziness. Am J Otol. 1998;19:76-81

14. Treleaven J. Dizziness Handicap Inventory (DHI). Aust J Physiother. 2006;52:67.

15. Knapstad MK, Nordahl SHG, Goplen FK. Clinical characteristics in patients with cervicogenic dizziness: A systematic review. Heal Sci Rep. 2019;2:e134.

16. Reiley AS, Vickory FM, Funderburg SE. How to diagnose cervicogenic dizziness. Arch Physiother. 2017;7:12.

17. Clendaniel RA, Landel R. Physical Therapy Management of Cervicogenic Dizziness. Vestibular Rehabilitation. Philadelphia, Pennsylvania j vest rehal. 2014;6:590-6.

18. Lee KS, Lee JH. Effect of maitland mobilization in cervical and thoracic spine and therapeutic exercise on functional impairment in individuals with chronic neck pain. J Phys Ther Sci. 2017;29:531-5.

19. Maitland GD. Principles of Techniques. Maitland's Vertebral Manipulation. London: Butterworth Heinemann. 2001;2:171-82.

20. Cassou B, Derriennic F, Monfort C. Chronic neck and shoulder pain, age, and working conditions: longitudinal results from a large random sample in France. Occup Env Med. 2002;59:537-44.

21. Brooks JX, Cullen KE. Early vestibular processing does not discriminate active from passive self-motion if there is a discrepancy between predicted and actual proprioceptive feedback. J Neurophysiol. 2014;111:2465-78.

22. Luan H, Gdowski MJ, Newlands SD. Convergence of vestibular and neck proprioceptive sensory signals in the cerebellar interpositus. J Neurosci. 2013;33:1198-210.

23. Brandt T, Huppert DA. New type of cervical vertigo: Head motion-induced spells in acute neck pain. Neurology. 2016;86:974-5.

24. Peng B. Cervical Vertigo: Historical Reviews and Advances. World Neurosurg. 2018;109:347-50. 